\title{
Investigating regional variation of respiratory infections in a general practice syndromic surveillance system
}

\section{Sue Smith ${ }^{1}$, Roger Morbey ${ }^{1}$, Simon de Lusignan ${ }^{2,3}$, Richard G. Pebody ${ }^{4}$, Gillian E. Smith ${ }^{1}$, Alex J. Elliot ${ }^{1}$}

${ }^{1}$ Real-time Syndromic Surveillance Team, Field Service, National Infection Service, Public Health England, Birmingham B3 2PW, UK

${ }^{2}$ Nuffield Department of Primary Care Health Sciences, University of Oxford, Oxford OX2 6GG, UK

${ }^{3}$ Royal College of General Practitioners Research and Surveillance Centre, London NW1 2FB, UK

${ }^{4}$ Immunisation and Countermeasures, National Infection Service, Public Health England, London NW9 5EQ, UK

Address correspondence to Sue Smith, E-mail: sue.smith@phe.gov.uk.

\begin{abstract}
Background Established surveillance systems can follow trends in community disease and illness over many years. However, within England there are known regional differences in healthcare utilisation, which can affect interpretation of trends. Here, we explore regional differences for a range of respiratory conditions using general practitioner (GP) consultation data.

Methods Daily data for respiratory conditions were extracted from a national GP surveillance system. Average daily GP consultation rates per 100000 registered patient population were calculated by each region of England and for each study year (2013-17). Consultation rates and incidence rate ratios were also calculated for each condition by deprivation quintile and by rural, urban, and conurbation groups.

Results Upper and lower respiratory tract infections and asthma were higher in the North and the Midlands than in London and the South, were highest in the most deprived groups and tended to be higher in more urban areas. Influenza-like illness was highest in the least deprived and rural areas.

Conclusions There are consistent differences in GP consultation rates across the English regions. This work has improved our understanding and interpretation of GP surveillance data at regional level and will guide more accurate public health messages.
\end{abstract}

Keywords asthma, human influenza, medical geography, primary healthcare, public health surveillance, respiratory tract infections

\section{Introduction}

In the United Kingdom (UK) general practice (family medicine) is the main route for patients to access the National Health Service (NHS). ${ }^{1}$ The role of primary care is to diagnose a wide range of health problems, treating illness in the community and managing long-term conditions in an increasingly complex population. General practice morbidity data have been an important indicator of community disease and illness, and established surveillance systems can follow trends over many years. Primary care surveillance systems such as the Royal College of General Practitioners (RCGP) Research and Surveillance Centre (RSC) are considered the 'gold standard' for primary care surveillance. ${ }^{2-4}$

Respiratory infections cause an annual burden on health services, particularly during the winter. ${ }^{5}$ General practitioner (GP) consultations, emergency department attendances, hos- pital admissions and deaths can all increase during the winter season, placing added pressures on different aspects of the health service. Public health surveillance systems play an important role in providing the intelligence needed to plan for and manage these pressures. Surveillance systems (e.g. laboratory testing) can monitor the appearance and frequency of the specific pathogens responsible for community respiratory infections and disease or monitor increases in the number

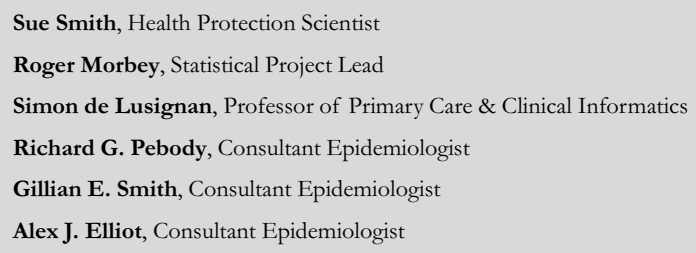


of symptomatic patients presenting to healthcare services. In particular, primary care data on respiratory infections form an important contribution to influenza surveillance in the UK where data are instrumental in determining the beginning of the 'influenza season', the intensity and peak of seasonal influenza activity. ${ }^{6}, 7$ Influenza has been shown to play a major role in winter pressures, ${ }^{8}$ and associated surveillance programmes have been developed to monitor and provide early warning of influenza activity. Public Health England (PHE) coordinates an influenza and respiratory virus programme which comprises a range of surveillance systems, including microbiological testing and syndromic surveillance. To establish the start, spread and severity of influenza activity, surveillance systems must be capable of analysing data by age group, and to analyse data to a regional and subregional level. Previous influenza outbreaks have illustrated the utility of this approach where localised hotspots of activity were the prelude to wide spread national activity. ${ }^{9}$

When routinely monitoring surveillance data across different regions, it is important to understand underlying regional differences in healthcare utilisation. England has long-term differences in health between the north and the south of the country. ${ }^{10}$ Previous work has also demonstrated regional variation in GP consultations for some respiratory conditions, which tend to be consistently higher in the north than in the south of England. ${ }^{11,} 12$ This has implications for comparing regional data as there are clear differences between regions, and it is important to understand those differences. Furthermore, GP data have been shown to vary by socioeconomic deprivation and by the urban rural environment in addition to potential differences in age structure between populations. $^{13,14}$ Deprivation and social determinants are a major cause of poor health, ${ }^{15,16}$ and it is therefore important to identify the key drivers of health inequalities to further our understanding of how to target and reduce these inequalities across healthcare services.

In order to fully understand regional differences in the presentation of respiratory disease, and account for these differences in surveillance outputs used to accurately describe trends and spread of disease, this paper aimed to explore the regional differences for a range of respiratory conditions using a GP surveillance system routinely used by PHE.

\section{Methods}

\section{Data sources and geography}

PHE maintains a suite of national syndromic surveillance systems that monitor a range of health data in real time. ${ }^{17}$ These data are aggregated into syndromic indicators based on the symptoms and clinical diagnoses of disease. For this study, we used daily data from a general practitioner (GP) 'in hours' (referring to the collection of data from GP services provided 'in hours', i.e. Monday to Friday normal working hours) syndromic surveillance system that collects anonymised data from a sample of GPs covering $\sim 49 \%$ of registered patients in England across the study period. Data for the respiratory conditions influenza-like illness (ILI), upper respiratory tract infection (URTI), lower respiratory tract infection (LRTI) and asthma were included in the study. ${ }^{18}$ The study period began in the first full year of data for the current system from 1 January 2013 to 31 December 2017, and data were stratified by age bands $<1$ year, 1-4 years, 5-14 years, 15-44 years, 45-64 years, 65-74 years and 75 years and over. Data were also analysed by English region using geographic boundaries of the nine PHE centres (the 'front door' for most of PHE's local services across health improvement, healthcare public health and health protection): North East, North West, Yorkshire and the Humber, East Midlands, West Midlands, East of England, London, South East and South West.

\section{Geographic analysis}

In order to identify regional differences and trends, an average daily GP consultation rate per 100000 registered patient population was calculated by region for each of the years in the study 2013-17. Weekends and public holidays, when GP practices are closed, were excluded from the rate calculation. GP consultation rates for each condition and region were age standardised, based on the 2016 England population, to adjust for demographic differences between the regions, and the data examined to see if differences between regions persisted.

\section{Statistical analysis}

To investigate the association between deprivation and consultations, a more granular geographical level was used, England's 152 upper tier local authorities (LA; a structure of local government). LAs were sorted in order of the English Index of Multiple Deprivation (IMD) rank and divided into quintiles. ${ }^{19}$ In order to remove possible age, confounding deprivation analysis was restricted to cases in the 15-64 years' age group. Age data at LA level was only available for a subset of data covering a population of 3.5 million patients. Data for the whole study period 2013-17 were included, with the exception of weekends and public holidays, where data for individual LAs were not available for one or more years during the study period the LA was excluded. Consultation rates and incidence rate ratios (IRRs) were calculated for each 
condition by IMD quintile using quintile 5 (least deprived) as a reference.

The same subset of 15-64 years' age group data was used to investigate whether the rural-urban status of an LA would affect the consultation rate. The Office of National Statistics rural-urban classification was used to divide the LAs into the six categories; then they were aggregated as follows: ${ }^{20}$ the mainly rural and largely rural formed the 'rural' group; the urban with significant rural, urban with city and town, and the urban with minor conurbation groups formed the 'urban' group; and the urban with major conurbation formed the 'conurbation' group. ${ }^{14}$ Rates and IRRs were calculated for each condition by rural, urban or conurbation group using the rural group as a reference.

\section{Results}

Average regional daily consultation rates per year of URTI ranged from 34.5 to 49.5 per 100000 population and were over twice as high as rates of LRTI (mean daily regional rate for each year 12.9-24.0 per 100 000). Rates of asthma were much lower being just over $4 \%$ of URTI rates (mean daily regional rate per year 1.5-2.6 per 100 000), while ILI rates were slightly lower (mean daily regional rate per year $0.8-1.7$ per 100 000).

In general, rates of URTI, LRTI and asthma were higher in the North and the Midlands than in London and the South. The North West consistently had the highest consultation rates for URTI (average mean daily regional rate per year (49.5 (range 47.8-51.1) per 100 000), LRTI (24.0 (23.2-24.6) per 100 000) and asthma (2.6 (2.4-2.7) per 100 000) (Fig. 1). By comparison, London had the lowest consultation rates for LRTI (12.9 (12.1-13.5) per 100 000) and asthma (1.5 (1.2-1.6) per 100 000). There was less of a consistent regional pattern for ILI consultation rates; however, there was a suggestion of a South to North gradient, with rates higher in southern regions and lower in the Midlands and North. London had the highest rate of ILI across the country.

Analysis of the data by socioeconomic and rural urban groups was carried out on a subset of cases in the 15-64 years' age group covering a population of 3.5 million patients. GP consultations for URTI were highest in the two most deprived groups, with patients in IMD quintiles 1 and 2 being, respectively, 1.33 and 1.16 times more likely to consult for URTI than patients in quintile 5 , the least deprived group (Table 1). Similarly, GP consultations for LRTI were also highest in quintiles 1 and 2, both 1.27 times more likely to consult a GP than patients in quintile 5. Rates of asthma consultations tended to increase with deprivation, with the highest rate ratios in this study seen in quintiles 1 and 2, respectively, 1.56 and 1.58 times more likely to visit a GP than quintile 5. By contrast, ILI rates were highest in the least deprived group, quintile 5.

Rates of respiratory consultations for URTI, LRTI and asthma increased from rural to urban areas, with the highest rates in the conurbation group (Table 2). Patients living in the conurbation were 1.3 times more likely to consult a GP for asthma than those living in rural areas. ILI patients in rural areas had the highest rates, with those in the urban and conurbation groups having 0.86 times the consultation rate of those in rural areas.

\section{Discussion}

\section{Main finding of this study}

This work has shown that there are persistent differences in GP consultation rates for respiratory diagnoses between regions of England. Age standardising the consultation rates accounted for regional bias in the age of the population, and therefore these differences are likely due to other factors. GP consultation rates for URTI, LRTI and asthma were consistently highest in the North West region. Rates also differed by the level of deprivation and urbanisation with consultations for respiratory conditions including URTI, LRTI and asthma highest in the most deprived groups and also tending to increase as areas become more urban, with the highest rates in the conurbations. Conversely, ILI was highest in the least deprived and in rural areas.

\section{What is already known on this topic}

Previous work has shown that the North of England has poorer health than other parts of England, and this is linked to socioeconomic disadvantage, with increasing deprivation leading to poorer health. ${ }^{21}$ It is known that socioeconomic factors influence GP consultation rates, and recent work has shown consultation rates for patients in the most deprived IMD quintile to be $18 \%$ higher than those in the least deprived

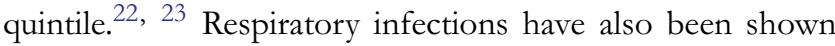
to be higher in the North, for example, a study of LRTI in older adults found that age-standardised incidence of LRTI was higher in the North and Midlands than in the South, with the highest rates in the North West, and also found that incidence increased with increasing deprivation. ${ }^{24}$ Work to develop intensity thresholds for influenza across the UK revealed that GP consultation rates for ILI varied across the devolved administrations of the UK, where each national sentinel surveillance system had a different pre-epidemic threshold to denote the start of the influenza season, with Scotland 


\section{Upper respiratory tract infection}

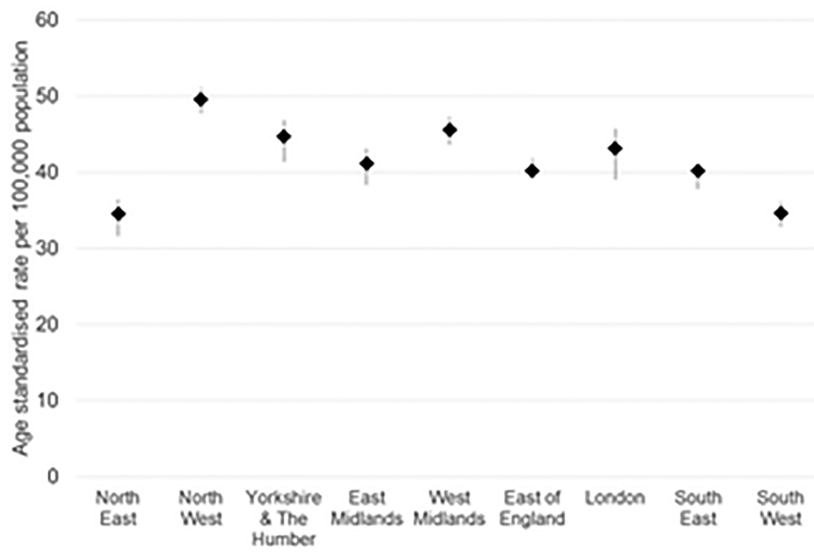

\section{Asthma}

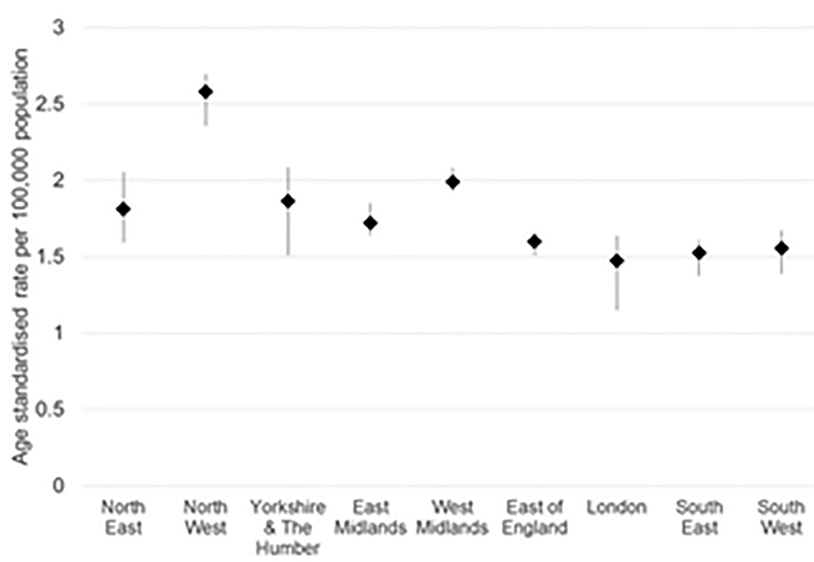

\section{Herpes zoster}

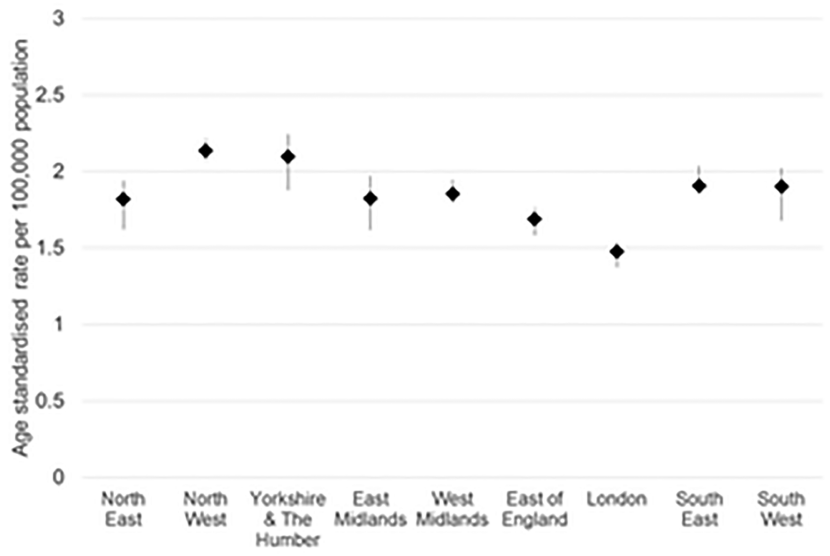

\section{Lower respiratory tract infection}

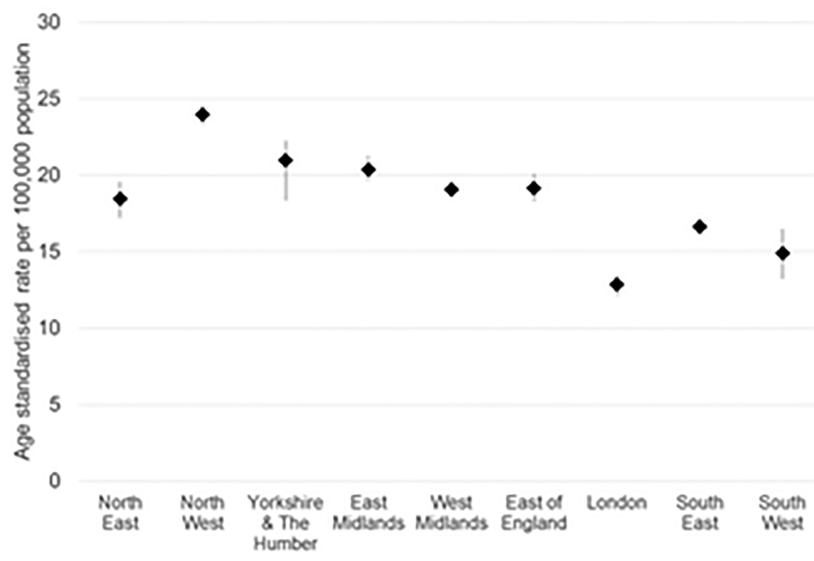

Influenza-like illness

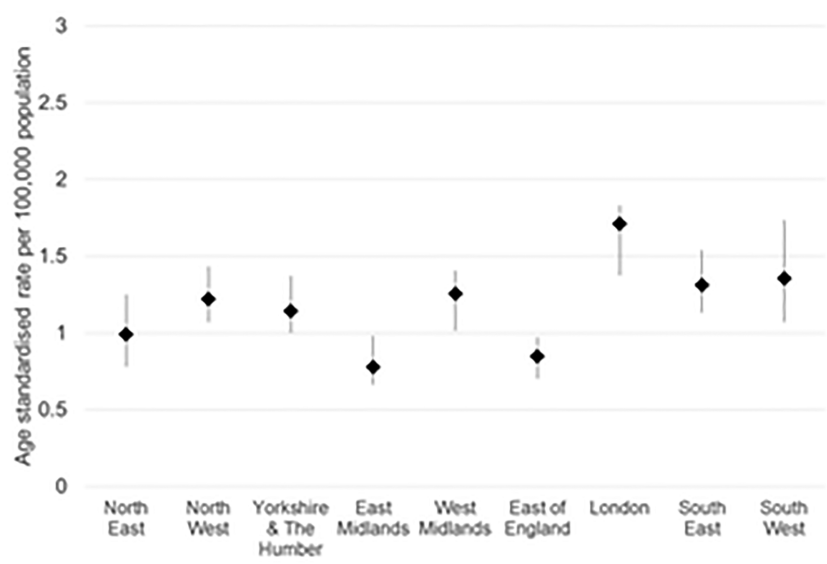

Fig. 1 Mean daily age standardised rate per 100000 population by region and showing range of mean values for each year 2013-17 (black diamond is the average of each year's mean).

having the highest threshold. ${ }^{25}$ The results from the current study support these findings, with highest rates reported in the North of England, and warrant further study of the spatial spread of ILI across the UK.
A recent study carried out on the RCGP Research and Surveillance Centre sentinel network of GP practices similarly found that those living in conurbations or urban areas were more likely to consult for URTI. ${ }^{14}$ However, the same study 
Table 1 Mean daily consultation rate (over the period 2013-17) per 100000 registered GP population (15-64 years) by Index of Multiple Deprivation (IMD) quintile

\begin{tabular}{|c|c|c|c|c|}
\hline \multirow{2}{*}{$\begin{array}{l}\text { Indicator } \\
\text { ILI }\end{array}$} & \multirow{2}{*}{$\frac{\text { IMD quintile }}{1}$} & \multirow{2}{*}{$\begin{array}{c}\begin{array}{c}\text { Rate per } 100000 \\
\text { population }\end{array} \\
0.47\end{array}$} & \multicolumn{2}{|c|}{ Rate ratio $(95 \% \mathrm{Cl})^{\mathrm{a}}$} \\
\hline & & & 0.89 & $(0.85-0.93)$ \\
\hline & 2 & 0.44 & 0.83 & $(0.79-0.87)$ \\
\hline & 3 & 0.41 & 0.78 & $(0.75-0.81)$ \\
\hline & 4 & 0.40 & 0.75 & $(0.72-0.78)$ \\
\hline & 5 & 0.53 & 1.00 & - \\
\hline \multirow[t]{5}{*}{ URTI } & 1 & 35.26 & 1.33 & $(1.33-1.34)$ \\
\hline & 2 & 30.74 & 1.16 & $(1.16-1.17)$ \\
\hline & 3 & 25.69 & 0.97 & $(0.97-0.98)$ \\
\hline & 4 & 26.48 & 1.00 & $(1.00-1.01)$ \\
\hline & 5 & 26.42 & 1.00 & - \\
\hline \multirow[t]{5}{*}{ LRTI } & 1 & 16.84 & 1.27 & $(1.26-1.28)$ \\
\hline & 2 & 16.88 & 1.27 & $(1.26-1.28)$ \\
\hline & 3 & 12.37 & 0.93 & $(0.92-0.94)$ \\
\hline & 4 & 13.90 & 1.05 & $(1.04-1.05)$ \\
\hline & 5 & 13.28 & 1.00 & - \\
\hline \multirow[t]{5}{*}{ Asthma } & 1 & 2.48 & 1.56 & $(1.53-1.60)$ \\
\hline & 2 & 2.51 & 1.58 & $(1.54-1.62)$ \\
\hline & 3 & 1.90 & 1.19 & $(1.17-1.22)$ \\
\hline & 4 & 1.63 & 1.02 & (1.00-1.05) \\
\hline & 5 & 1.59 & 1.00 & - \\
\hline \multirow[t]{5}{*}{ Herpes zoster } & 1 & 1.15 & 0.87 & $(0.85-0.90)$ \\
\hline & 2 & 1.22 & 0.92 & $(0.89-0.95)$ \\
\hline & 3 & 1.04 & 0.79 & $(0.77-0.81)$ \\
\hline & 4 & 1.15 & 0.87 & $0.85-0.89)$ \\
\hline & 5 & 1.32 & 1.00 & - \\
\hline
\end{tabular}

aStatistically significant (at 95\% confidence level) results are in bold. ILI, influenza-like illness; URTI, upper respiratory tract infection; LRTI, lower respiratory tract infection and $\mathrm{Cl}$, confidence interval.

found that those in rural areas had an increased risk of asthma and LRTI. This contrasts with our finding that the conurbation group were most likely to consult for LRTI and asthma. In the RCGP study, the age range was different, attribution of rurality and deprivation were at the individual level and there was a greater proportion of older people living in the rural areas, who are more likely to consult for LRTI; however, the reason for the increased asthma risk was unclear. The study looked at all age groups while our comparison of rural, urban and conurbation groups was confined to the 1564 years' age group to remove the effects of demographic differences between the groups so these differences are likely to be due to the different age distributions studied.

\section{What this study adds}

The GP data used in this study are from a large representative dataset of healthcare seeking behaviour in a network of community-based GPs in England. This is therefore a unique opportunity to investigate a large cross-section of the population, and this adds to the evidence base highlighting inequalities observed in healthcare seeking behaviour for different respiratory infections (both acute and chronic) across England. This study revealed consistent differences in patients presenting to primary care health services with these conditions. Reducing health inequalities is currently a public health priority, and therefore this evidence further highlighting their existence supports public health programmes that aim to reduce and end these inequalities. ${ }^{26,27}$

The GP in hours syndromic surveillance system contributes to national influenza surveillance, national syndromic surveillance, and also local surveillance bulletins produced by PHE centres, as it covers around half of the England population and so is able to report at a national, regional and subregional level. The results of this study will provide a greater understanding of the differences between regional 
Table 2 Mean daily consultation rate (over the period 2013-17) per 100000 registered GP population (15-64 years) by rural, urban and conurbation

\begin{tabular}{|c|c|c|c|c|}
\hline \multirow{2}{*}{$\begin{array}{l}\text { Indicator } \\
\text { ILI }\end{array}$} & \multirow{2}{*}{$\begin{array}{c}\text { Rural urban } \\
\text { Rural }\end{array}$} & \multirow{2}{*}{$\begin{array}{c}\begin{array}{c}\text { Rate per } 100000 \\
\text { population }\end{array} \\
0.50\end{array}$} & \multicolumn{2}{|c|}{ Rate ratio $(95 \% \mathrm{Cl})^{\mathrm{a}}$} \\
\hline & & & 1.00 & - \\
\hline & Urban & 0.43 & 0.86 & (0.83-0.89) \\
\hline & Conurbation & 0.43 & 0.86 & $(0.83-0.89)$ \\
\hline \multirow[t]{3}{*}{ URTI } & Rural & 24.66 & 1.00 & - \\
\hline & Urban & 28.10 & 1.14 & $(1.13-1.14)$ \\
\hline & Conurbation & 30.98 & 1.26 & $(1.25-1.26)$ \\
\hline \multirow[t]{3}{*}{ LRTI } & Rural & 12.95 & 1.00 & - \\
\hline & Urban & 14.33 & 1.11 & (1.10-1.11) \\
\hline & Conurbation & 14.88 & 1.15 & $(1.14-1.16)$ \\
\hline \multirow[t]{3}{*}{ Asthma } & Rural & 1.75 & 1.00 & - \\
\hline & Urban & 1.82 & 1.04 & $(1.02-1.06)$ \\
\hline & Conurbation & 2.26 & 1.30 & $(1.27-1.32)$ \\
\hline \multirow[t]{3}{*}{ Herpes zoster } & Rural & 1.31 & 1.00 & - \\
\hline & Urban & 1.13 & 0.86 & $(0.84-0.88)$ \\
\hline & Conurbation & 1.13 & 0.86 & $(0.84-0.88)$ \\
\hline
\end{tabular}

a Statistically significant (at 95\% confidence level) results are in bold. ILI, influenza-like illness; URTI, upper respiratory tract infection; LRTI, lower respiratory tract infection and $\mathrm{Cl}$, confidence interval.

rates for respiratory conditions and the reasons for those differences.

It is important to be aware of these persistent regional differences when comparing GP consultation rates since a rate that is lower than that of the other regions could potentially provide false reassurance. Similarly, in a region such as the North West, particularly high rates of respiratory illness may not be unusual for that area. Within the PHE syndromic surveillance service, the statistical method employed to identify unusual activity uses a multilevel mixed effects negative binomial regression model (RAMMIE) to fit historical daily syndromic data counts and provide estimates for current counts at a local, regional and national level in England. ${ }^{28}$ The model accounts for day of the week/month effects and also adjusts for bank holidays (which can impact on healthcare consulting behaviour). RAMMIE estimates are used to create thresholds to generate statistical alarms whenever daily counts exceed the threshold. RAMMIE takes regional variations in activity into account to provide a more accurate assessment of whether or not a change is unusual and requires additional public health action. However, the statistical approach to interpreting syndromic surveillance data is only a part of this service; the epidemiological analysis and description of trends is an important component of this service, for which background knowledge of regional variations is critical to the accurate interpretation of data.
Analysis by deprivation and by rural urban groups has shown that respiratory consultations tend to be highest in the most deprived areas and in large conurbations. Asthma in particular is associated with deprivation and the conurbation. This may be due to the known higher levels of air pollution in city areas. There is scope for better public health messaging to help address these issues particularly when there is a known increase in pollution levels.

There was an opposite gradient of association between ILI and deprivation compared to the other respiratory indicators. ILI is, in general, an acute self-limiting infection compared to LRTI and asthma, which include more chronic disease. There is an association between higher incidence of chronic respiratory disease and areas of high deprivation. ${ }^{29}$ It is therefore possible that in deprived areas, where a higher proportion of patients present to GP services with chronic respiratory disease, symptoms of ILI are more likely to be coded within a chronic respiratory disease diagnostic coding group; in less deprived areas with lower rates of chronic disease, ILI might be recorded uniquely as a code for ILI.

\section{Limitations of this study}

The PHE GP in hours surveillance system has good coverage of the England population; however participation by GP practices is voluntary, so coverage can vary across the country depending on the numbers of practices that contribute to 
the system. Only two of the main providers of practice information systems contribute to the system which also limits coverage, although between them they cover $90 \%$ of the England population. ${ }^{30}$ While age group data are available down to regional level across the system, only limited age group data are available at LA level so analysis at this level can only be done on a subset of the data. The most granular level of geographical data available in the system is upper tier LA, and data can only be analysed by location of GP practice rather than by patient residence, both of which affect the accuracy of assignment of IMD quintiles and the rural urban classification.

There is no case definition for the conditions reported, they are instead defined by groups of diagnostic codes. Careful consideration must be given to the choice of codes to include in the code lists as omission of a commonly used code could give an underestimate of cases. If a condition is miscoded, for example, influenza vaccination coded as influenza, this misclassification may not be picked up by the system.

While we have accounted for the effects of urban, rural and deprivation status, there are other factors that we have not considered in this study, for example, variation in the uptake of flu vaccine. ${ }^{31}$ Other possible variables contributing to the associations may include geographical differences in coding practice, environmental factors (including climate, pollen and spores) and patient healthcare seeking behaviour: each of these is worthy of further exploration.

We have shown consistent differences in consultation rates across the English regions and explored some of the reasons for this variation. This work will enable us to improve our understanding of GP syndromic surveillance at a regional level and help to guide public health action.

\section{Acknowledgements}

We thank the PHE Real-Time Syndromic Surveillance Team for technical expertise. We acknowledge support from TPP and participating SystmOne practices and University of Nottingham, ClinRisk, EMIS Health and EMIS practices submitting data to the QSurveillance database.

\section{Funding}

This work was supported by the National Institute for Health Research (NIHR) Health Protection Research Unit (HPRU) in Emergency Preparedness and Response [AJE, RAM and GES]. The views expressed are those of the author(s) and not necessarily those of the NHS, the NIHR and the Department of Health and Social Care or Public Health England.

\section{Conflicts of interest}

SdeL reports funding from Public Health England through RCGP to support the RCGP Research and Surveillance Centre; through the University of Surrey, he has received grants from GSK, Seqirus and Takeda. All other authors report no potential conflicts.

\section{References}

1 Marshall M. A precious jewel: the role of general practice in the English NHS. N Engl J Med 2015;372:893-7.

2 Fleming DM, Elliot AJ. Lessons from 40 years' surveillance of influenza in England and Wales. Epidemiol Infect 2008;136:866-75.

3 de Lusignan S, Correa A, Smith GE, et al. RCGP research and surveillance Centre: 50 years' surveillance of influenza, infections, and respiratory conditions. BrJ Gen Pract 2017;67:440-1.

4 de Lusignan S, Correa A, Pebody R, et al. Incidence of lower respiratory tract infections and atopic conditions in boys and young male adults: Royal College of general practitioners research and surveillance Centre annual report 2015-2016. JMIR Public Health Surveill 2018;e49:4.

5 Morbey RA, Elliot AJ, Harcourt S, et al. Estimating the burden on general practitioner services in England from increases in respiratory disease associated with seasonal respiratory pathogen activity. Epidemiol Infect 2018;146:1389-96.

6 Public Health England. Weekly national flu reports: 2018 to 2019 season. 2018. https://www.gov.uk/government/statistics/weekly-na tional-flu-reports-2018-to-2019-season (13 November 2018, date last accessed)

7 Public Health England. Sources of UK fu data: influenza surveillance in the UK. 2018. https://www.gov.uk/guidance/sources-of-uk-flu-data-i nfluenza-surveillance-in-the-uk\#clinical-surveillance-through-prima ry-care (15 November 2018, date last accessed).

8 Pebody RG, Green HK, Warburton F, et al. Significant spike in excess mortality in England in winter 2014/15 - influenza the likely culprit. Epidemiol Infect 2018;146:1106-13.

9 Smith S, Smith GE, Olowokure B, et al. Early spread of the 2009 influenza $\mathrm{A}(\mathrm{H} 1 \mathrm{~N} 1)$ pandemic in the United Kingdom: use of local syndromic data, May-August 2009. Euro Surveill 2011;16(3):pii=19771. https://doi.org/10.2807/ese.16.03.19771-en.

10 Buchan IE, Kontopantelis E, Sperrin M, et al. North-south disparities in English mortality1965-2015: longitudinal population study. J Epidemiol Commun Health 2017;71:928-36.

11 Smith S, Smith GE, Heatlie $\mathrm{H}$, et al. Reducing variation in antibacterial prescribing rates for 'cough/cold' and sore throat between 1993 and 2001: regional analyses using the general practice research database. Public Health 2006;120:752-9.

12 Simpson CR, Hippisley-Cox J, Sheikh A. Trends in the epidemiology of chronic obstructive pulmonary disease in England: a national study of 51804 patients. BrJ Gen Pract 2010;60:277-84.

13 Todkill D, Loveridge P, Elliot AJ, et al. Socioeconomic and geographical variation in general practitioner consultations for allergic rhinitis in England, 2003-2014: an observational study. BMJ Open 2017;7:e017038. 
14 de Lusignan S, McGee C, Webb R, et al. Conurbation, urban, and rural living as determinants of allergies and infectious diseases: Royal College of general practitioners research and surveillance Centre annual report 2016-2017. JMIR Public Health Surveill 2018;4:e11354.

15 Hawker JI, Olowokure B, Sufi F, et al. Social deprivation and hospital admission for respiratory infection: an ecological study. Respir Med 2003;97:1219-24.

16 Marmot M, Allen J, Goldblatt P, et al. Fair society, health lives: strategic review of health inequalities in England. 2010. http://www.instituteofhea lthequity.org/resources-reports/fair-society-healthy-lives-the-ma rmot-review (22 October 2019, date last accessed).

17 Elliot AJ, Morbey RA, Hughes HE, et al. Syndromic surveillance: a public health legacy of the London 2012 Olympic and Paralympic games. Public Health 2013;127:777-81.

18 Fleming DM, Bartelds A, Chapman RS, et al. The consistency of shingles and its significance for health monitoring. Eur J Epidemiol 2004;19:1113-8.

19 Department for Communities and Local Government. The indices of deprivation. 2015. https://www.gov.uk/government/statistics/engli sh-indices-of-deprivation-2015 (22 October 2019, date last accessed).

20 Department for Environment Food and Rural Affairs. 2011 Rural urban classification of local authorities and other geographies. 2016. https:// www.gov.uk/government/statistics/2011-rural-urban-classificatio n-of-local-authority-and-other-higher-level-geographies-for-statisti cal-purposes (22 October 2019, date last accessed).

21 Whitehead M, Bambra C, Barr B, et al. Due North: the report of the inquiry on health equity for the North 2014. https://www.gmcvo.org.u k/system/files/Due-North-Report-of-the-Inquiry-on-Health-Equi ty-in-the-North-final.pdf (22 October 2019, date last accessed).

22 Carr-Hill RA, Rice N, Roland M. Socioeconomic determinants of rates of consultation in general practice based on fourth national morbidity survey of general practices. BMJ 1996;312:1008-12.
23 Mukhtar TK, Bankhead C, Stevens S, et al. Factors associated with consultation rates in general practice in England, 2013-2014: a crosssectional study. BrJ Gen Pract 2018;68:e370-e7.

24 Millett ER, Quint JK, Smeeth L, et al. Incidence of communityacquired lower respiratory tract infections and pneumonia among older adults in the United Kingdom: a population-based study. PLoS One 2013;8:e75131.

25 Green HK, Charlett A, Moran-Gilad J, et al. Harmonizing influenza primary-care surveillance in the United Kingdom: piloting two methods to assess the timing and intensity of the seasonal epidemic across several general practice-based surveillance schemes. Epidemiol Infect 2015;143:1-12.

26 Public Health England. PHE Strategy 2020 to 2025. 2019. https:// www.gov.uk/government/publications/phe-strategy-2020-to-2025 (22 October 2019, date last accessed).

27 CDC. Strategies for reducing health disparities - selected CDCsponsored intervention, United States, 2016. MMWR Suppl 2016;65:1-69.

28 Morbey RA, Elliot AJ, Charlett A, et al. The application of a novel 'rising activity, multi-level mixed effects, indicator emphasis' (RAMMIE) method for syndromic surveillance in England. Bioinformatics 2015;31:3660-5.

29 Anto JM, Vermeire P, Vestbo J, et al. Epidemiology of chronic obstructive pulmonary disease. Eur Respir J 2001;17:982-94.

30 Kontopantelis E, Stevens RJ, Helms PJ, et al. Spatial distribution of clinical computer systems in primary care in England in 2016 and implications for primary care electronic medical record databases: a cross-sectional population study. BMJ Open 2018;8:e020738.

31 Tessier E, Warburton F, Tsang C, et al. Population-level factors predicting variation in influenza vaccine uptake among adults and young children in England, 2015/16 and 2016/17. Vaccine 2018;36: 3231-8. 